Relations industrielles

Industrial Relations

\title{
Gagnon, Robert P., Le droit du travail du Québec: théories et pratiques
}

\section{Rodrigue Blouin}

Volume 50, numéro 2, 1995

URI : https://id.erudit.org/iderudit/051022ar

DOI : https://doi.org/10.7202/051022ar

Aller au sommaire du numéro

Éditeur(s)

Département des relations industrielles de l'Université Laval

ISSN

0034-379X (imprimé)

1703-8138 (numérique)

Découvrir la revue

Citer ce compte rendu

Blouin, R. (1995). Compte rendu de [Gagnon, Robert P., Le droit du travail du Québec: théories et pratiques]. Relations industrielles / Industrial Relations, 50(2), 451-453. https://doi.org/10.7202/051022ar

Tous droits réservés (C) Département des relations industrielles de l'Université Laval, 1995
Ce document est protégé par la loi sur le droit d'auteur. L'utilisation des services d'Érudit (y compris la reproduction) est assujettie à sa politique d'utilisation que vous pouvez consulter en ligne.

https://apropos.erudit.org/fr/usagers/politique-dutilisation/ 
that the labour movement should not kowtow to legal restrictions on strike activity because to do so only entrenches labour's subordinate position.

Of course, the loss of mid-contract strike rights is counterbalanced, many would argue, by the legislatively mandated grievance arbitration process. Drache and Glasbeek, in chapter seven, make several very good points about why this tradeoff is not always quite what it seems. While accepting that grievance arbitration has limited some capricious employer actions, nevertheless, it must be recognized that arbitration is slow, expensive, and the outcomes are often uncertain. Drache and Glasbeek particularly question some longstanding arbitral principles - the "obey now, grieve later" rule and the notion that employer discipline stands until set aside. In this latter regard, the authors propose that the system be turned on end; disciplinary sanctions would not be implemented until they passed muster before an arbitrator.

In the final four chapters, the authors focus their attention on broader macroeconomic concerns. In these chapters they discuss systemic problems such as the female wage gap, unemployment, inadequate pension systems and unreasonably dangerous work environments. The authors note that while unionized workers have made some (very modest) gains in these areas, unorganized workers, who comprise the bulk of the Canadian workforce, are even worse off. What is to be done? The authors support broad-based (read: private sector) pay equity programs; mandatory indexing of both private and public pension programs, stricter enforcement of existing safety regulations, granting the right to strike (not the mere individual right to refuse to work) over unsafe work conditions, enriched welfare programs, and an end to free-trade.

Unfortunately, the authors give little, if any, guidance about how their costly agenda might be sold to the public in times in fiscal crisis, other than to suggest that corporations pay higher taxes. This is, I believe, the major failing of the book. It is one thing to make recommendations for reform, however, it is incumbent on those who propose new public expenditures to put a realistic dollar estimate forward as well as a reasonable financing plan. Otherwise, and with good reason, a reform proposal is unlikely to be given any serious consideration. I strongly suspect that this book will not be a catalyst to any "reshaping" of our industrial relations system. And that is a pity as the system is in need of reform.

KENNETH WM. THORNICROFT University of Victoria

\section{Le droit du travail du Québec: théories et pratiques ( $2^{e}$ édition) par Robert P. GaGnoN, Cowansville, Les Éditions Yvon Blais, 1993, 611 p., ISBN 2-89073-899-X.}

Cette excellente synthèse descriptive sur l'état du droit du travail en vigueur décevra néanmoins le lecteur qui aurait apprécié une seconde édition plus critique que la première. L'évolution jurisprudentielle et certaines récentes modifications à la législation soulèvent en effet de nombreuses interrogations qui auraient appelé des mises en situation plus élaborées avant de préciser les sens et portée d'une règle; l'auteur passe trop rapidement.

L'approche retenue et le plan de travail adopté expliquent en bonne partie cette absence d'intérêt pour l'analyse critique. Ainsi l'ouvrage répond à une 
orientation purement classique sur la façon d'appréhender le droit du travail. Outre un titre préliminaire qui fait un rappel des grands principes régissant l'application du droit constitutionnel en matière de législation du travail ainsi que des chartes des droits et libertés, l'ouvrage s'attache d'abord au champ des rapports individuels de travail puis à celui des rapports collectifs. Cette division masque à peine une présentation par "blocs législatifs" plutôt que selon une compréhension qui intègre en un tout cohérent l'ensemble des dispositions de droit du travail. Il s'ensuit que les diverses pièces législatives sont étudiées indépendamment les unes des autres, quitte à faire quelques observations ponctuelles pour les rattacher à un même tronc commun.

En raison de cette méthode, l'auteur se voit contraint de ramener sous le titre des rapports individuels de travail l'étude des normes du travail et celles concernant la santé et la sécurité du travail. Pourtant les normes d'ordre public s'imposent non seulement en contexte d'une relation individuelle de travail mais aussi lors de tout rapport collectif, tout comme d'ailleurs les lois prééminentes que l'auteur présente au titre préliminaire. Il aurait été avantageux de regrouper en un même titre l'ensemble des prescriptions s'imposant d'autorité à toute forme de rapport de travail. Un tel regroupement aurait permis d'attirer l'attention du praticien sur certains épineux problèmes qui risquent de le confronter dans un avenir rapproché. Ainsi sont escamotées les modalités d'application du principe qui veut que la législation prééminente et d'ordre public s'impose d'autorité au contrat individuel de travail, à la convention collective ordinaire ou extensionnée ou encore, à tout document (par exemple, le règlement d'entreprise). L'auteur en discute certes mais de façon ponctuelle. Une approche plus systémique lui aurait permis d'aborder notamment la question de la hiérarchie des sources juridiques ainsi que celle de l'application de la norme la plus avantageuse, deux délicats problèmes avec lesquels les tribunaux du travail sont de plus en plus confrontés. De même, une présentation plus intégrée aurait facilité la discussion sur les interrelations entre les domaines des rapports statutaires, individuels et collectifs du travail, plus particulièrement à la lumière de la disposition préliminaire du Code civil du Québec qui édicte que dans les matières de droit commun, ce code "constitue le fondement des autres lois qui peuvent ellesmêmes ajouter au code ou y déroger ". Un exposé plus systémique aurait aussi permis de mettre en relief l'émergence d'une notion d'entreprise et les conséquences qui en résultent en pratique, à la lumière des articles 2097 du Code civil du Québec, 45 et 46 du Code du travail ainsi que 96 et 97 de la Loi sur les normes du travail. On peut de même regretter l'absence d'essai pour situer les droits et obligations du salarié en lien d'emploi précaire ou atypique. Enfin, il nous apparaît que l'analyse des dispositions législatives en termes de régimes juridiques plutôt que par pièces législatives aurait mieux répondu aux besoins de l'enseignement et de la pratique en contexte contemporain (par exemple, régime juridique de la rémunération, régime juridique des bénéfices et avantages sociaux, etc.).

Ces critiques s'adressent avant tout au choix de l'auteur de respecter l'approche classique trop souvent retenue dans l'enseignement du droit du travail. L'ouvrage demeure par ailleurs d'une excellente qualité dans les matières traitées. Le style de l'auteur est clair et précis. Les opinions émises sont tranchées et appuyées de nombreuses références; les causes rapportées sont d'ailleurs rappelées en une table de la jurisprudence et il s'agit d'un précieux instrument de travail.

Le titre préliminaire, consacré aux aspects constitutionnels et quasi constitutionnels, permet de se sensibiliser à 
des donnés qui n'appartiennent pas en propre au champ du droit du travail mais dont la connaissance est dans une certaine mesure nécessaire. Bien que bref (36 p.), l'exposé comprend l'essentiel pour savoir s'il faut entreprendre une étude plus fondamentale sur l'un ou l'autre des aspects traités.

Le contrat de travail (61 p.), les normes de travail ( 41 p.) et la santé et sécurité du travail (2I p.) sont étudiés en autant de chapitres distincts au titre premier. L'importance accordée au contrat individuel de travail s'explique indéniablement par la récente réforme de ce contrat. L'analyse est menée rondement. On notera deux points d'intérêt : d'une part, l'étude du concept d'entreprise (p. 84-88) alors que l'auteur y fait ressortir que la vente en justice n'est pas exclue contrairement au Code du travail et à la Loi sur les normes du travail; d'autre part, les observations faites à propos des aspects fiscaux qu'il sied de prendre en considération lors d'une rupture illégale ou injustifiée du lien d'emploi alors que l'auteur est d'avis que ceux-ci doivent être pris en considération pour la détermination des dommages-intérêts (p. 95-97). Les deux autres chapitres témoignent d'une belle synthèse de la Loi sur les normes du travail et de la Loi sur la santé et la sécurité au travail. L'auteur n'y vise que l'essentiel. Sa méthode sera plus particulièrement appréciée de l'étudiant et du praticien qui en sont à leurs premières armes en droit des rapports individuels de travail ou encore, qui en sont élojgnés depuis quelque temps. Les nombreuses références permettent par ailleurs d'approfondir les questions litigieuses abordées.

Le second titre, intitulé les rapports collectifs du travail, ne vise que le régime général (Code du travail) et non pas les régimes d'exception (cons- truction et Sûreté du Québec). En quelque 300 pages, l'auteur y étudie succes. sivement, en huit chapitres distincts, le champ d'application et l'administration du Code, le droit d'association, la procédure en accréditation, la vie de l'accréditation, la négociation collective et l'arbitrage des différends, les conflits de négociation (grève, lock-out, piquetage), la convention collective et, finalement, l'arbitrage des griefs. L'auteur est manifestement à l'aise avec ces matières. L'analyse est menée rapidement et avec précision. Le lecteur y trouve une mine d'informations jurisprudentielles sur l'état du droit en vigueur. On notera plus particulièrement une analyse bien nourrie portant sur le statut de salarié au sens du Code (p. 170-179) et l'observation fondée voulant que "la jurisprudence du Tribunal du travail apparaît plutôt restrictive ". Il faut aussi signaler les propos consacrés à la transmission d'entreprise. Ceux-ci témoignent de la difficulté de ce sujet qui a donné lieu à " une longue saga judiciaire " ( $\mathrm{p}$. 319). Ils permettent néanmoins d'obtenir une bonne vue d'ensemble sur les orientations jurisprudentielles en rapports collectifs. En faisant le pont avec les observations faites sur ce mēme concept en contexte des rapports individuels, le lecteur peut s'imprégner d'une vue plus globale et comparative.

Au bilan de l'analyse il faut regretter la structure classique de l'ouvrage. Par contre la description de l'état du droit saura satisfaire pleinement l'étudiant ou le praticien en quête d'informations techniques utiles pour arrêter une position en regard d'une question litigieuse. Les notes infrapaginales constituent à cet égard une excellente source de renseignements.

RODRIGUE BLOUIN Université Laval 